\begin{tabular}{|c|c|}
\hline Title & Growth and characterization of wurtzite InP A IG aP core-multishell nanowires with $\mathrm{A} I \mathrm{G}$ aP quantum well structures \\
\hline Author(s) & Ishizaka, Fumiya; Hiraya, Y oshihiro; Tomioka, Katsuhiro; Motohisa, Junichi; Fukui, Takashi \\
\hline Citation & $\begin{array}{l}\text { Japanese Journal of A pplied Physics, 56(1), } 010311 \\
\text { https://doi.org/10.7567/JJA P.56.010311 }\end{array}$ \\
\hline Issue Date & 2017-01 \\
\hline Doc URL & http:/hdl.handle.net/2115/68025 \\
\hline Rights & (C) 2017 The Japan Society of A pplied Physics \\
\hline Type & article (author version) \\
\hline File Information & 161026_WZA IGaP_JJA PRC_manuscript.pdf \\
\hline
\end{tabular}

Instructions for use 


\title{
Growth and characterization of wurtzite InP/AIGaP core-multishell nanowires with AlGaP quantum well structures
}

Fumiya Ishizaka ${ }^{1}$, Yoshihiro Hiraya ${ }^{1}$, Katsuhiro Tomioka ${ }^{1,2}$, Junichi Motohisa ${ }^{1}$, and Takashi Fukui $^{1}$

${ }^{1}$ Graduate School of Information Science and Technology, and Research Center for Integrated Quantum Electronics (RCIQE), Hokkaido University, North 13 West 8, Sapporo 060-8628, Japan

2 PRESTO, Japan Science and Technology Agency (JST), Kawaguchi, Saitama 332-0012, Japan

E-mail: ishizaka@rciqe.hokudai.ac.jp

\begin{abstract}
We report on selective-area growth and characterization of wurtzite (WZ) InP/AlGaP core-multishell nanowires. Quantum well (QW) structures were fabricated in the AlGaP multishells by changing alloy compositions. Transmission electron microscopy revealed that the AlGaP multishell was grown with a WZ structure on side of the WZ InP core. The lattice constants of the $\mathrm{WZ}$ InP core and WZ AlGaP shell were determined by X-ray diffraction. Cathodoluminescence studies showed that the WZ AlGaP QW with an $\mathrm{Al}$ composition of $20 \%$ had green emissions at $2.37 \mathrm{eV}$. These results open the possibility for green light-emitting diodes using WZ AlGaP based materials.
\end{abstract}


Color mixed RGB light-emitting diodes (LEDs) are promising candidates for future solidstate lightning and display technology due to their potential advantages in energy conversion efficiency and color rendering index (CRI). ${ }^{1,2)}$ However, achieving high efficient LEDs in the green region is challenging due to a lack of suitable semiconductor materials, which is known as the "green gap".3,4) Non-nitride III-V materials having wurtzite (WZ) structures have recently offered a new approach to overcome this issue. Some theories predict that GaP and AlP in the WZ structures have direct band gaps in contrast to the conventional zinc blende (ZB) structures with indirect band gaps. ${ }^{5-7)}$ Although this approach has been experimentally demonstrated in $\mathrm{WZ} \mathrm{GaP}^{8,9)}$ and $\mathrm{WZ} \mathrm{AlInP}{ }^{10)}$ these $\mathrm{WZ}$ materials with quantum well (QW) structures required for LED applications have not been reported so far. In nanowire (NW) structures, radial core-multishell (CMS) NWs with QW structures are suitable structures for LED applications. ${ }^{11,12)}$ In this study, we report the growth and characterization of WZ InP/AlGaP CMS NWs with QW structures for the green color spectrum. The WZ AlGaP at an $\mathrm{Al}$ composition of around $20 \%$ is expected to have the band gap energy in the green spectral region according to calculations of their band structures. ${ }^{6,7)}$

The CMS NWs were synthesized by selective-area metal organic vapor phase epitaxy (SA-MOVPE). In SA-MOVPE, InP NWs can be grown with a pure WZ structure by properly adjusting the growth conditions. ${ }^{13-15)}$ Based on these WZ InP NWs, the crystal structure transfer method ${ }^{16-18)}$ was applied for the radial multishell growth. At first, a 20-nm-thick $\mathrm{SiO}_{2}$ layer was deposited on an InP (111)A substrate using plasma sputtering, and the $\mathrm{SiO}_{2}$ layer was partially removed using electron-beam (EB) lithography and wet chemical etching. The $\mathrm{SiO}_{2}$ patterns were designed to be a periodic array of openings with a diameter of $130 \mathrm{~nm}$. The SA-MOVPE was performed in a low-pressure MOVPE reactor using trimethylaluminum (TMAl), trimethylgallium (TMGa), trimethylindium (TMIn), and tertiarybutylphosphine 
(TBP) as source materials. Prior to the growth, the native oxide on the openings was removed using thermal cleaning for $5 \mathrm{~min}$ at $600^{\circ} \mathrm{C}$ under a hydrogen and TBP ambient. After thermal cleaning, WZ InP NWs were grown for $15 \mathrm{~min}$ at $660^{\circ} \mathrm{C}$ with a V/III ratio of 18 , which is the typical condition for growing WZ structures. ${ }^{13)}$ The partial pressures of TMIn and TBP were $2.7 \times 10^{-6}$ and $4.9 \times 10^{-5}$ atm, respectively. Low temperature (LT) AlGaP shells were then grown for $7 \mathrm{~min}$ at $600^{\circ} \mathrm{C}$ with a V/III ratio of 650 to prevent InP NWs from P desorptions. The respective partial pressures of TMAl, TMGa, and TBP were $2.4 \times 10^{-7}, 2.4 \times 10^{-7}$, and $3.1 \times 10^{-4} \mathrm{~atm}$, which correspond to the TMAl supply ratio, [TMAl]/[TMAl]+[TMGa], of 0.50 in the vapor phase. After LT AlGaP shell growth, three layers of AlGaP shells were grown to fabricate QW structures by changing the TMAl supply ratios. The inner barrier layer was grown for 20 min at $750^{\circ} \mathrm{C}$ with a V/III ratio of 320 . The respective partial pressures of TMAl, TMGa, and TBP were $4.8 \times 10^{-7}, 4.8 \times 10^{-7}$, and $3.1 \times 10^{-4}$ atm, which correspond to the TMAl supply ratio of 0.50 in the vapor phase. The QW layer was grown for $3 \mathrm{~min}$ at $700^{\circ} \mathrm{C}$ with a V/III ratio of 320. The respective partial pressures of TMAl, TMGa, and TBP were $1.9 \times 10^{-7}, 7.7 \times 10^{-7}$, and $3.1 \times 10^{-4} \mathrm{~atm}$, which correspond to the TMAl supply ratio of $0.20 \mathrm{in}$ the vapor phase. The outer barrier layer was grown for $10 \mathrm{~min}$ at $700^{\circ} \mathrm{C}$ with a V/III ratio of 320. The partial pressures of TMAl, TMIn, and TBP were the same as those of the inner barrier layer. Finally, the GaP shell was grown for $1 \mathrm{~min}$ at $700^{\circ} \mathrm{C}$ with a V/III ratio of 650 as a capping layer to avoid oxidation of AlGaP. The partial pressures of TMGa and TBP were $9.6 \times 10^{-7}$ and $6.2 \times 10^{-4}$ atm, respectively.

The morphology, crystal structures, and compositions of CMS NWs were characterized using a scanning electron microscope (SEM), transmission electron microscope (TEM), and energy dispersive X-ray spectroscopy (EDX) combined with a scanning transmission electron microscope (STEM). The electron-beam projection was parallel to the $<-110>$ direction. Xray diffraction (XRD) measurements were carried out to investigate the crystal structures of 
the NW assembly. The NW pitch was $1600 \mathrm{~nm}$ and investigated sample area was about 4 $\mathrm{mm}^{2}$, where more than one million NWs were included. Cathodoluminescence (CL) measurements were performed at $34 \mathrm{~K}$ on a single CMS NW with an acceleration voltage of $7 \mathrm{kV}$. Based on the calculation by Kanaya et al., ${ }^{19)}$ the penetration depth of the electrons was about $500 \mathrm{~nm}$ under this condition, whereas most of the electrons were generated within a fraction of the penetration depth.

An SEM image and a schematic illustration of InP/AlGaP CMS NWs with QW structures are shown in Figs. 1(a) and 1(b). The NWs had a hexagonal pillar shape with $\{-110\}$ side facets. The NW pitch was $600 \mathrm{~nm}$. The average height and total diameter of the CMS NWs were $2.6 \mu \mathrm{m}$ and $240 \mathrm{~nm}$, respectively. The slightly larger diameter was found at the tip of the NWs, which corresponds to the AlGaP shell grown on top of the InP core. From the SEM image, tapering and bending of the NWs were not observed, while poly-crystal depositions on $\mathrm{SiO}_{2}$ films were observed. These depositions began to appear when the AlGaP shell growth time was 20 min or more.

To ensure QW structures fabricated in the AlGaP shell, we investigated the compositions of the CMS NWs using EDX measurements. A high angle annular dark field (HAADF) STEM image of the NW cross-section in the middle part and corresponding EDX mappings are shown in Figs. 2(a)-2(e). From these images, the diameter of the InP core and the thickness of the AlGaP shell were approximately 125 and $50 \mathrm{~nm}$, respectively. The QW thickness was about $3 \mathrm{~nm}$. The two barrier layers had different thicknesses because the growth time of the outer barrier layer was shorter than that of the inner barrier layer. This is also because the growth rate of the outer AlGaP shell decreased as the growth time increased probably due to the increasing depositions of poly-crystals on the $\mathrm{SiO}_{2}$ mask area. 
Figure 2(a) shows two types of black contrasts. The black contrast around the InP core resulted from the low crystal quality of the LT AlGaP shell layer. The other black contrast along the <-211> radial directions was aluminum segregation, which commonly appears in aluminum-containing shell layers, such as GaAs/AlInP core-shell NWs. ${ }^{20)}$ Aluminum segregation was also found in EDX mappings in Figs. 2(d) and 2(e). In addition to aluminum segregation, indium diffusion appeared in the AlGaP shell, which can be seen in both the EDX mappings and line scan in Figs. 2(b) and 2(f). The reason for this indium diffusion is that the growth temperature of the AlGaP shell is higher than that of the InP core. From Fig. 2(f), the aluminum and gallium compositions in the barrier layers were almost the same excluding the effect of indium diffusion, which is equal to the supply ratio of TMAl and TMGa. Therefore, the aluminum and gallium compositions in the QW layer were estimated to be $20 \%$ and $80 \%$, respectively.

To ensure the crystal structures of a CMS NW, we performed TEM and fast-Fouriertransform (FFT) analyses on a NW, and these are shown in Fig. 3. Three parts of the NW were investigated. First, the InP core had a WZ structure with a few stacking faults per micrometer. Second, the AlGaP shell on top of the InP core had a ZB structure with stacking faults. In Fig. 3(a), the diameter in the top part of the NW was larger than that in the middle part. This offers another piece of evidence that the AlGaP shell on the top consists of $\mathrm{ZB}$ structures because stacking faults and lateral growth continuously occur until stable $\{-110\}$ facets are formed in case of selective area growth of NWs with ZB structures. ${ }^{14,21)}$ Third, the AlGaP shell on the side of the InP core mainly had a WZ structure, but it partly had a ZB structure resulting from stacking faults. The appearance ratio of $\mathrm{WZ}$ segments in the AlGaP shell was calculated using the statistical analysis ${ }^{22)}$ to be $84 \%$. We consider the stacking faults and ZB segments appeared at the interface of the InP core and AlGaP shell for the 
following reasons. For planar layers, the critical thickness is calculated to be less than $1 \mathrm{~nm}$ from the lattice mismatch of $7.0 \%$ between InP and $\mathrm{Al}_{0.5} \mathrm{Ga}_{0.5} \mathrm{P}$. When the AlGaP shell is grown on the InP core, it can be coherently grown on the InP core until it reaches the critical thickness, and then misfit dislocations appear in the AlGaP shell. As a result, additional layers are introduced in $\mathrm{WZ}$ layers of the AlGaP shell that has a smaller lattice constant, resulting in $\mathrm{ZB}$ segments such as “ABABCBCB...” and “ABACABAB...”.

Figure 4 shows an XRD reciprocal space mapping (RSM) around the ZB (331) reflection of the InP (111)A substrate. The WZ (10-15) and ZB (331) peaks were observed for the InP core and substrate, and the WZ (10-15), ZB (224) twin, and faint ZB (331) peaks were observed for the AlGaP shell. The strong intensity of the WZ InP (10-15) peak indicates that the InP core has a high crystal quality in the WZ structure. In contrast, the intensity of the WZ AlGaP (10-15) peak was relatively weak and broad due to a low crystal quality resulting from stacking faults. The ZB (224) twin peak and faint spot of ZB (331) for AlGaP were attributed to the $\mathrm{ZB} \mathrm{AlGaP}$ shell with rotational twins grown on the top of the $\mathrm{InP}$ core because the ZB (224) twin peak was not observed for both the InP core and substrate. These results are in good agreement with the TEM analysis.

Next, we measured axial and radial lattice constants, $c_{W Z}$ and $a_{W Z}$, of the WZ InP core and WZ AlGaP shell from the peak positions of WZ (10-15) reflections, and compared the measured values with relaxed WZ lattice constants geometrically converted from ZB lattice constants. For converted values, the relations $a_{W Z}=1 / \sqrt{2} a_{Z B}$ and $c_{W Z}=\sqrt{8 / 3} a_{W Z}$ were used, and Vegard's law was applied to the calculations of AlGaP lattice constants. The calculation results were summarized in Table I. For WZ InP, both the measured $a_{W Z}$ and $c_{W Z}$ values were almost the same as the converted $a_{W Z}$ and $c_{W Z}$ values. Kriegner et al. have reported that free standing WZ InP is elongated in the $c$-axis direction and compressed in the $a$-axis direction as compared to the converted values. ${ }^{23)}$ These tendencies were also found in other III-V 
NWs. ${ }^{24,25)}$ However, in this study, such a tendency was not found probably because the WZ InP core was surrounded by an $\mathrm{AlGaP}$ shell that has a smaller lattice constant and was therefore subject to compressive strains in the $c$-axis direction. For WZ AlGaP, both the measured $a_{W Z}$ and $c_{W Z}$ values were substantially larger than the converted $a_{W Z}$ and $c_{W Z}$ values. This implies the incorporation of InP that has a larger lattice constant. The measured lattice constants of WZ AlGaP correspond to those with indium incorporation of about $10 \%$ when they are geometrically converted using Vegard's law. This result is consistent with the EDX analysis discussed in Fig. 2.

To understand the effects of WZ and ZB structures on optical properties, we carried out CL measurements on a single NW at $34 \mathrm{~K}$. The electron beam was scanned along the NW axis with 16 measured points at intervals of about $150 \mathrm{~nm}$ as shown in Fig. 5(a). The obtained CL spectra are shown in Fig. 5(b). For clarity in CL peak energies, representative CL spectra at the top and middle of the NW are depicted in Fig. 5(c) using a logarithmic scale. The peaks at 1.42 and $1.62 \mathrm{eV}$ are emissions from the ZB InP substrate and $\mathrm{WZ}$ InP core NW. The peaks at 1.36-1.38 eV observed in beam positions \#15 and 16 in Fig. 5(b) correspond to bound exciton and donor-acceptor emissions of the InP substrate. ${ }^{26)}$ The peak energy for ZB InP is equivalent to the known value, whereas the peak energy for WZ InP is slightly higher than those previously reported. ${ }^{13)}$ The band gap energy of $\mathrm{WZ} \mathrm{InP}$ is $1.502 \mathrm{eV}$ for the theoretical value, and the band gap energy of previously reported WZ InP NWs is $1.54 \mathrm{eV}$. In this study, the WZ InP core has compressive strain arising from the AlGaP shell, which can lead to the increase in the band gap energy. If the band gap energy of the WZ InP NWs is the same as that of previously reported ones, the effect of compressive strain will lead to an energy shift of $80 \mathrm{meV}$. 
Regarding the AlGaP multishell, CL emissions were observed at 2.18 and $2.37 \mathrm{eV}$. From the electron beam spot, the former can be attributed to $\mathrm{ZB} \mathrm{AlGaP}$ on the top of the InP core, and the latter can be attributed to $\mathrm{WZ} \mathrm{AlGaP}$ on the side. We consider that these two emissions were originated from the AlGaP QWs because we found in a similar material combination that simple core-shell NWs without QW structures had no CL emissions from the shells. Although the origin of the peak at $2.30 \mathrm{eV}$ observed in beam positions \#3-5 in Fig. 5(b) was unclear, it was probably related to WZ/ZB type II junctions at the interface of WZ and $\mathrm{ZB} \mathrm{AlGaP}$ shells. The reason for the low emission intensity was that the generated electron-hole pairs can easily be moved from the AlGaP shell to the InP core. The results indicate that green emissions at $2.37 \mathrm{eV}(523 \mathrm{~nm})$ were evident from the WZ AlGaP QW, which had a shorter wavelength than reported ones. ${ }^{8,10)}$

The estimated band gap for $\mathrm{WZ} \mathrm{Al}_{0.2} \mathrm{Ga}_{0.8} \mathrm{P}$ is $2.394 \mathrm{eV}$ using a linear approximation from the values found in the literature. ${ }^{6,27)}$ The $\mathrm{WZ} \mathrm{Al}_{0.5} \mathrm{Ga}_{0.5} \mathrm{P} / \mathrm{Al}_{0.2} \mathrm{Ga}_{0.8} \mathrm{P} / \mathrm{Al}_{0.5} \mathrm{Ga}_{0.5} \mathrm{P} \mathrm{QW}$ is predicted to be type I band alignment with the conduction and valence band offsets of 93 and $112 \mathrm{meV}$, and the quantum confinement effect of 3-nm-thick QW leads to an energy blue shift of $111 \mathrm{meV}$. In addition, the effect of indium incorporation of 10\%, which was suggested by XRD and EDX measurements, leads to an energy red shift of $92 \mathrm{meV}$. Therefore, the emission energy of our WZ QW is estimated to be $2.413 \mathrm{eV}$. This estimation is consistent with the experimental result. Calculation based on a ZB QW gave similar confinement energy and red shift due to In incorporation, thus, the ZB QW is less likely. However, discussing the nature of these emissions in more detail is difficult at present and consideration of strain effect and the variations in compositions and QW thicknesses should be required for more accurate assessment. In this regard, further optical investigations such as time-resolved measurements are required to clarify whether or not they have direct band gaps. 
In summary, we investigated the growth morphology, composition, and structural and optical properties of $\mathrm{WZ}$ InP/AlGaP CMS NWs grown using the crystal structure transfer method. Cross-sectional STEM and EDX studies showed that the QW structures were formed in the AlGaP multishell. TEM analysis revealed that the InP core had a WZ structure with very few stacking faults and that the AlGaP multishell was grown with a WZ structure on the side of the InP core, while it was grown with a ZB structure on the top. From XRD measurements, the measured lattice constants of $\mathrm{WZ} \mathrm{InP} \mathrm{were} \mathrm{almost} \mathrm{the} \mathrm{same} \mathrm{as} \mathrm{the}$ calculated values, while those of WZ AlGaP were larger due to indium outdiffusion. Finally, we found green emissions at $2.37 \mathrm{eV}$ from the $\mathrm{WZ}$ AlGaP QW and showed that the difference in the crystal structures affects CL emission energies in the AlGaP QWs. Our results demonstrate a pathway for green LEDs using WZ AlGaP based materials.

\section{Acknowledgements}

The authors would like to thank Professor Shinjiro Hara and Dr. Keitaro Ikejiri for their valuable advice as well as Dr. Masatoshi Yoshimura, Dr. Eiji Nakai, and Mr. Muyi Chen for their support with the experiments. This work was financially supported by a Grant-in-Aid for Scientific Research (S) (KAKENHI Grant Number 23221007) provided by the Japan Society for the Promotion of Science (JSPS). 


\section{References}

1) J. Y. Tsao, M. E. Coltrin, M. H. Crawford, and J. A. Simmons, Proc. IEEE 98, 1162 (2010).

2) Y. H. Ra, R. Wang, S. Y. Woo, M. Djavid, S. Sadaf, J. Lee, G. A. Botton, and Z. Mi, Nano Lett. 16, 4608 (2016).

3) M. R. Krames, O. B. Shchekin, R. Mueller-Mach, G. O. Mueller, L. Zhou, G. Harbers, and M. G. Craford, J. Disp. Technol. 3, 160 (2007).

4) M. Auf der Maur, A. Pecchia, G. Penazzi, W. Rodrigues, and A. Di Carlo, Phys. Rev. Lett. 116, 027401 (2016).

5) M. Murayama and T. Nakayama, Phys. Rev. B 49, 4710 (1994).

6) A. De and C. E. Pryor, Phys. Rev. B 81, 155210 (2010).

7) F. Bechstedt and A. Belabbes, J. Phys.: Condens. Matter 25, 273201 (2013).

8) S. Assali, I. Zardo, S. Plissard, D. Kriegner, M. A. Verheijen, G. Bauer, A. Meijerink, A. Belabbes, F. Bechstedt, J. E. M. Haverkort, and E. P. A. M. Bakkers, Nano Lett. 13, 1559 (2013).

9) A. Berg, S. Lehmann, N. Vainorius, A. Gustafsson, M-E. Pistol, L. R. Wallenberg, L. Samuelson, and M. T. Borgström, J. Cryst. Growth 386, 47 (2014).

10) Y. Hiraya, F. Ishizaka, K. Tomioka, and T. Fukui, Appl. Phys. Express 9, 035502 (2016).

11) K. Tomioka, J. Motohisa, S. Hara, K. Hiruma, and T. Fukui, Nano Lett. 10, 1639 (2010).

12) A. Berg, S. Yazdi, A. Nowzari, K. Storm, V. Jain, N. Vainorius, L. Samuelson, J. B. Wagner, and M. T. Borgstrom, Nano Lett. 16, 656 (2016).

13) Y. Kitauchi, Y. Kobayashi, K. Tomioka, S. Hara, K. Hiruma, T. Fukui, and J. Motohisa, Nano Lett. 10, 1699 (2010). 
14) K. Ikejiri, Y. Kitauchi, K. Tomioka, J. Motohisa, and T. Fukui, Nano Lett. 11, 4314 (2011).

15) Q. Gao, D. Saxena, F. Wang, L. Fu, S. Mokkapati, Y. Guo, L. Li, J. W.-Leung, P. Caroff, H. H. Tan, and C. Jagadish, Nano Lett. 14, 5206 (2014).

16) R. E. Algra, M. Hocevar, M. A. Verheijen, I. Zardo, G. G. W. Immink, W. J. P. van Enckevort, G. Abstreiter, L. P. Kouwenhoven, E. Vlieg, and E. P. A. M. Bakkers, Nano Lett. 11, 1690 (2011).

17) F. Ishizaka, Y. Hiraya, K. Tomioka, and T. Fukui, J. Cryst. Growth 411, 71 (2015).

18) M. Heurlin, T. Stankevič, S. Mickevičius, S. Yngman, D. Lindgren, A. Mikkelsen, R. Feidenhans’l, M. T. Borgström, L. Samuelson, Nano Lett. 15, 2462 (2015).

19) K. Kanaya and S. Okayama, J. Phys. D: Appl. Phys. 5, 43 (1972).

20) J. B. Wagner, N. Sköld, L. R. Wallenberg, and L. Samuelson, J. Cryst. Growth 312, 1755 (2010).

21) K. Ikejiri, T. Sato, H. Yoshida, K. Hiruma, J. Motohisa, S. Hara, and T. Fukui, Nanotechnology 19, 265604 (2008).

22) K. Tomioka, J. Motohisa, S. Hara, and T. Fukui, Jpn. J. Appl. Phys. 46, L1102 (2007).

23) D. Kriegner, E. Wintersberger, K. Kawaguchi, J. Wallentin, M. T. Borgström, and J. Stangl, Nanotechnology 22, 425704 (2011).

24) D. Kriegner, C. Panse, B. Mandl, K. A. Dick, M. Keplinger, J. M. Persson, P. Caroff, D. Ercolani, L. Sorba, F. Bechstedt, and J. Stangl, G. Bauer, Nano Lett. 11, 1483 (2011).

25) D. Kriegner, S. Assali, A. Belabbes, T. Etzelstorfer, V. Holý, T. Schülli, F. Bechstedt, E. P. A. M. Bakkers, G. Bauer, and J. Stangl, Phys. Rev. B 88, 115315 (2013).

26) K. Kuriyama, K. Ushiyama, T. Tsunoda, M. Uchida, and K. Yokoyama, J. Electron. Mater. 27, 462 (1998). 
27) I. Vurgaftman, J. R. Meyer, and L. R. Ram-Mohan, J. Appl. Phys. 89, 5815 (2001). 


\section{Figure captions}

Fig. 1. (Color online) (a) $30^{\circ}$-tilted view SEM image of InP/AlGaP CMS NWs. (b) Schematic illustration of grown layers of the CMS NW.

Fig. 2. (Color online) (a) Cross-sectional HAADF STEM image of a CMS NW and corresponding EDX mappings of (b) In, (c) P, (d) Al, and (e) Ga, showing the existence of a QW structure. (f) EDX line scan of a CMS NW along the lateral direction and corresponding HAADF STEM image (inset).

Fig. 3. (Color online) Cross-sectional TEM images of an InP/AlGaP CMS NW. The incident of the electron beam was parallel to the $<-110>$ direction of the substrate. (a) Overall TEM image of the NW. (b) and (c) Enlarged TEM images at the top and side of the NW shown in (a). (d)-(i) High-resolution TEM images and corresponding FFT images of the top AlGaP shell, side AlGaP shell, and InP core.

Fig. 4. (Color online) XRD RSM of InP/AlGaP CMS NWs recorded around the ZB (331) reflection of the $\operatorname{InP}(111)$ A substrate.

Fig. 5. (Color online) CL spectra of a single InP/AlGaP CMS NW measured at $34 \mathrm{~K}$. The electron beam was scanned along the NW axis. (a) Measured points and (b) corresponding CL spectra of the NW. (c) Representative CL spectra at the top and middle of the NW shown in a logarithmic scale. 
Table I. WZ lattice constants of the InP core and AlGaP shell.

\begin{tabular}{lllll}
\hline \multicolumn{1}{c}{ Material } & \multicolumn{1}{c}{ Method } & $a(\AA)$ & $c(\AA)$ & $c / a$ \\
\hline WZ InP & Conversion & 4.1498 & 6.7766 & 1.633 \\
& Experimental $^{\mathrm{a}}$ & 4.1423 & 6.8013 & 1.642 \\
& This work & 4.15 & 6.77 & 1.63 \\
$\mathrm{WZ} \mathrm{Al}_{0.5} \mathrm{Ga}_{0.5} \mathrm{P}$ & Conversion & 3.8587 & 6.3012 & 1.633 \\
& This work & 3.93 & 6.43 & 1.64 \\
\hline
\end{tabular}

${ }^{\mathrm{a}}$ From Ref. 23. 
Fig. 1

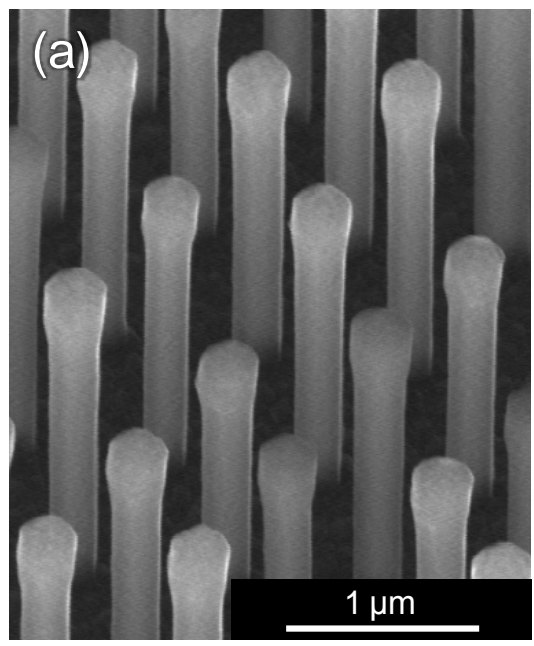

(b)

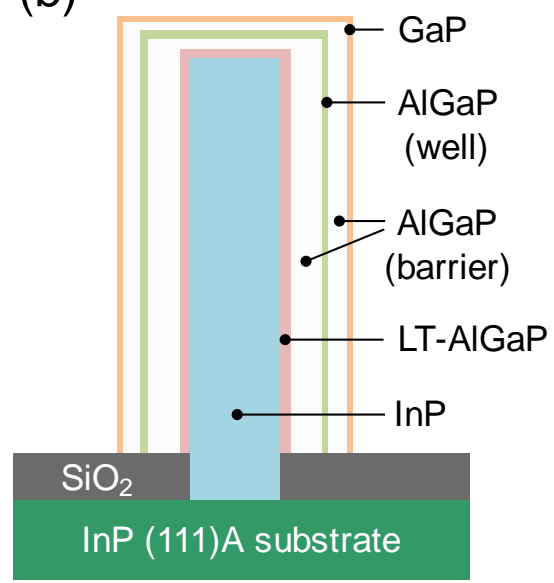


Fig. 2
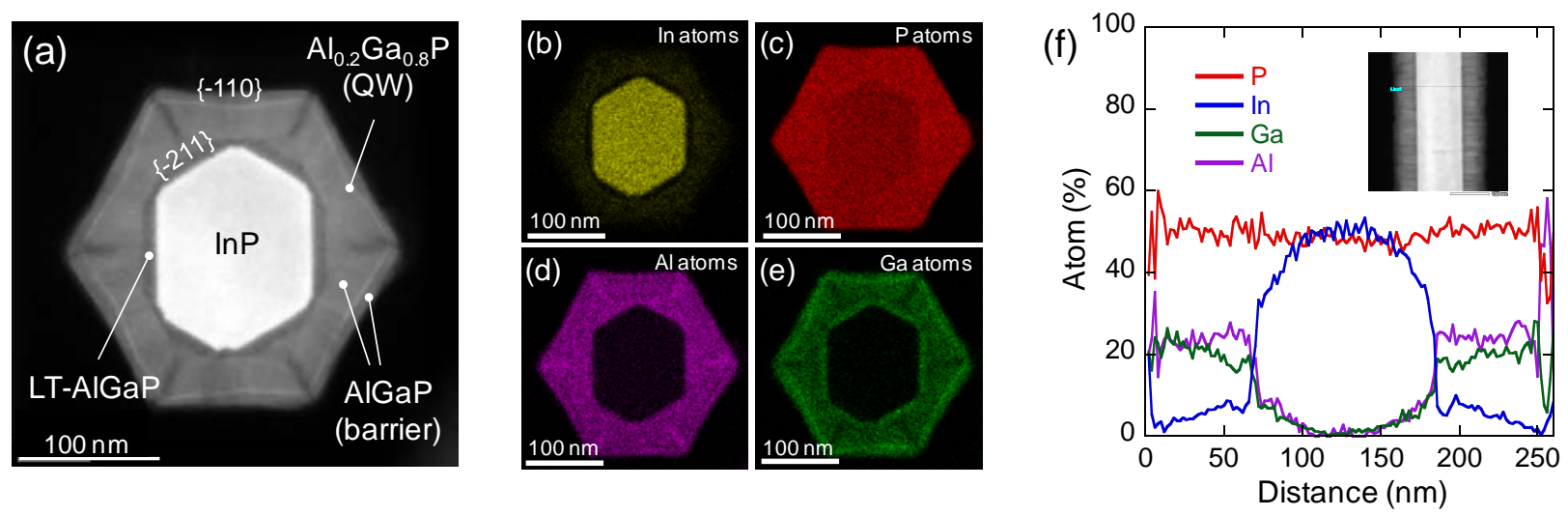
Fig. 3
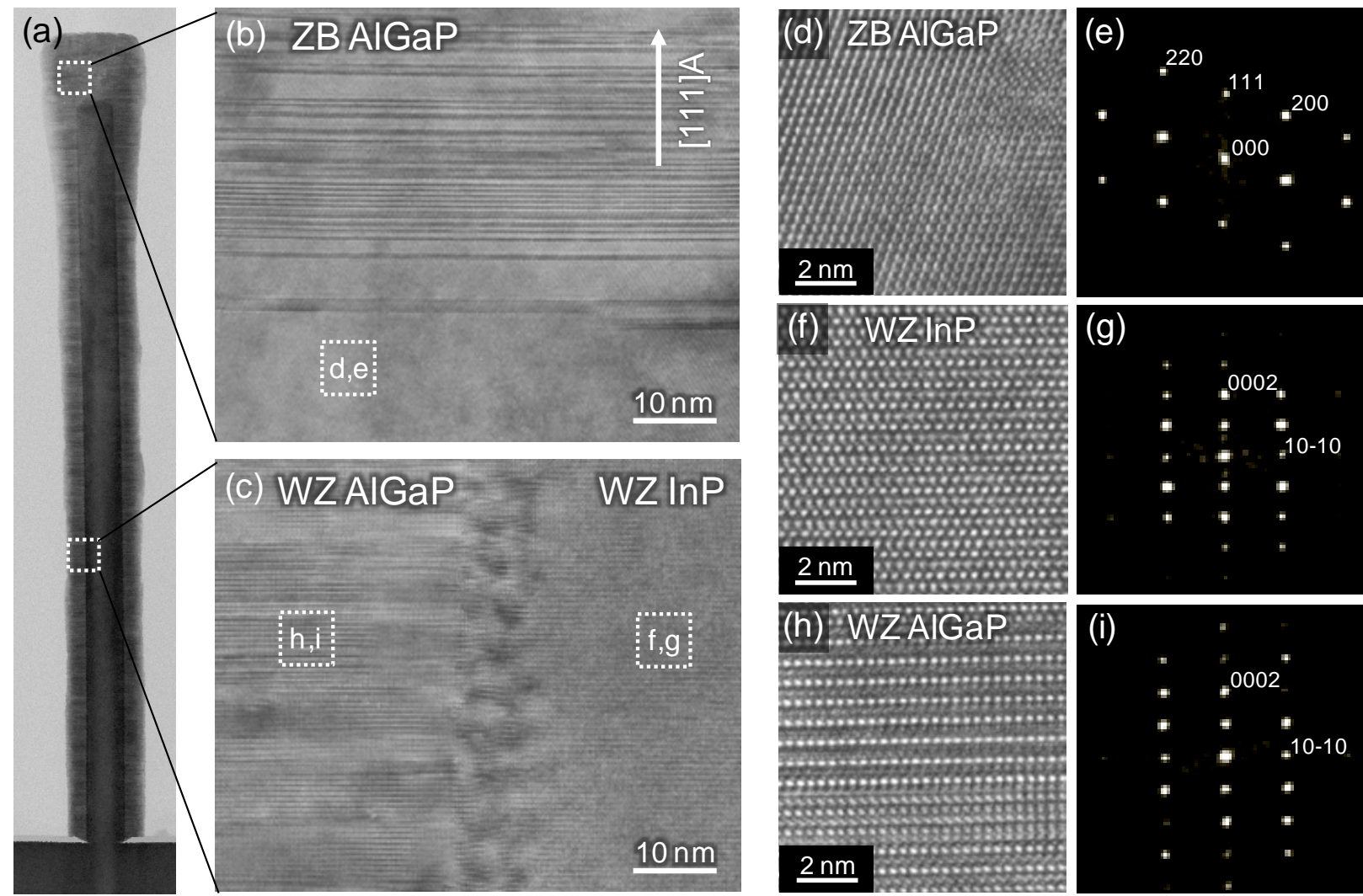
Fig. 4

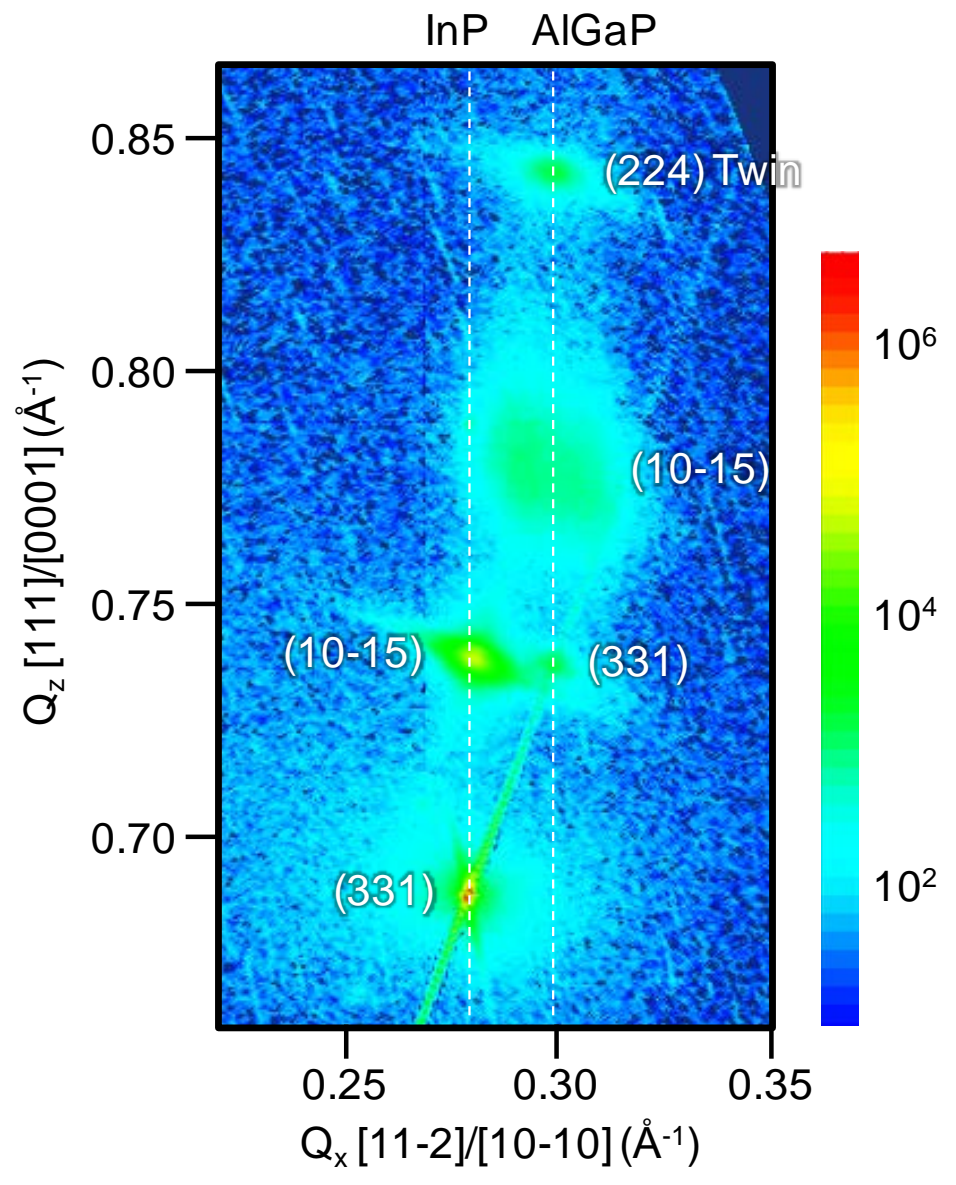


Fig. 5
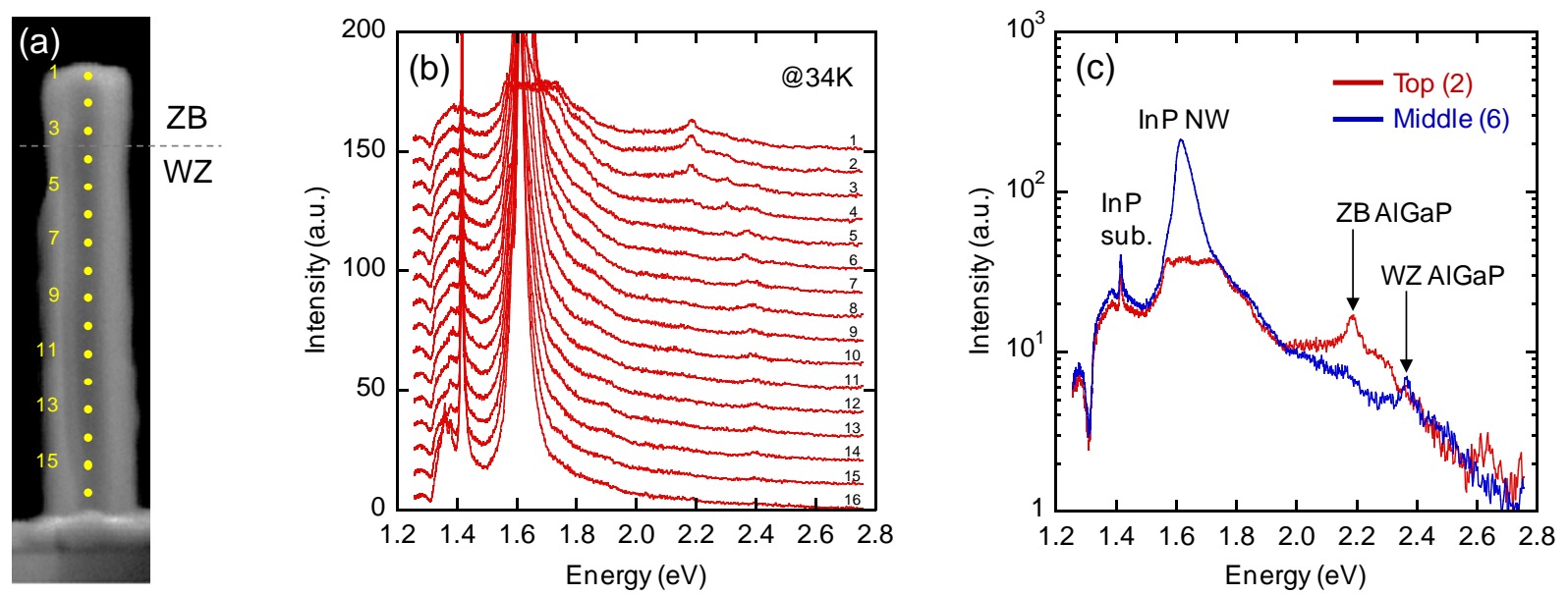